\title{
Sound radiation of a railway rail in close proximity to
}

\section{the ground}

\author{
Xianying Zhang ${ }^{1 *}$, Giacomo Squicciarini ${ }^{1}$, David J Thompson ${ }^{1}$ \\ ${ }^{1}$ Institute of Sound and Vibration Research, University of Southampton, Southampton SO17 \\ 1BJ, UK \\ * Corresponding author address: xianyingzhang96@ gmail.com
}

\begin{abstract}
The sound radiation of a railway in close to proximity to a ground (both rigid and absorptive) is predicted by the boundary element method (BEM) in two dimensions (2D). Results are given in terms of the radiation ratio for both vertical and lateral motion of the rail, when the effects of the acoustic boundary conditions due to the sleepers and ballast are taken into account in the numerical models. Allowance is made for the effect of wave propagation along the rail by applying a correction in the $2 \mathrm{D}$ modelling. It is shown that the $2 \mathrm{D}$ correction is necessary at low frequency, for both vertical and lateral motion of an unsupported rail, especially in the vicinity of the corresponding critical frequency. However, this correction is not applicable for a supported rail; for vertical motion no correction is needed to the $2 \mathrm{D}$ result while for lateral motion the corresponding correction would depend on the pad stiffness. Finally, the corresponding numerical predictions of the sound radiation from a rail are verified by comparison with experimental results obtained using a $1 / 5$ scale rail model in different configurations.
\end{abstract}




\section{Introduction}

Railways are generally viewed as an environmentally-friendly and sustainable means of transportation. However, noise and vibration often lead to adverse reactions to both new and existing railways. The most important source of environmental noise from railways is rolling noise. It is produced by vibration of the track and wheel, which is induced by their combined surface roughness at the wheel/rail contact [1]. The relative importance of the wheel and track radiation depends on details of their design, the roughness spectra and the train speed [1]. Nonetheless, the component of noise radiated by the rail is often the highest, and dominates the important mid-frequency region between 400 and $2000 \mathrm{~Hz}$. It is therefore necessary to understand the mechanisms of sound radiation by railway track in order to implement effective noise control measures.

Comprehensive analytical formulas were proposed by Remington [2] to predict wheel/rail rolling noise in urban rail transit systems based on the characterisation of the wheel/rail dynamic system developed in [3]. The results agreed well with measured wayside noise. For the sound radiation from the rail, using the model of Bender and Remington [4], the radiation efficiency was based on the uniform motion of simple cylindrical beams for both vertical and horizontal motion with an assumption that the rail was located in free field. Later, this theoretical analysis to predict wheel/rail rolling noise was expanded [5]. In particular, the noise propagation was estimated by modelling the ground as a finite impedance plane using the approach of Chessell [6]. Also, an extensive series of field measurements on a full scale urban rail transit vehicle was carried out [7] for comparison with the prediction of the wayside noise during a train passby by using the theoretical model in [5]. 
Thompson extended these engineering methods for predicting rolling noise [8] and a software package, known as TWINS ('Track-Wheel Interaction Noise Software'), was produced [9][10]. For the sound radiation from the rail in the TWINS model, a multiple source model based on the geometry of the rail cross-section was used to represent the vibrating rail [11]. One of the assumptions in this model, as in Bender and Remington [4], was that the rail radiation could be modelled in a free field. Similar results were obtained by using a two-dimensional boundary element model of the rail radiation in free space [12]. Comparisons with a three-dimensional model were also presented in [12] showing that a two-dimensional model gives satisfactory results in most practical cases for frequencies above about $250 \mathrm{~Hz}$. Below this frequency, allowance should be made for the high decay rate of vibration along the rail which means that the radiation resembles that from a point source instead of a line source.

Nilsson et al. [13] used a combination of waveguide finite elements and wavenumber boundary elements to calculate the sound radiation from a rail including an embedded rail as well as a rail in free space. Recently, the directivity of noise radiated by a rail has also been investigated by several authors [14][15][16].

The TWINS model for rolling noise has been validated by means of field measurements during train passby [9][10][17]. This showed reasonably good agreement between measurements and predictions over a wide range of conditions. Particularly where the noise was predicted directly from the measured vibration the agreement was good; larger uncertainty was found in the prediction of the vibration from the surface roughness.

In practice, the rail is not located in free space but is in close proximity to the ground. This consists of ballast, which is partially absorptive, and sleepers (or cross ties) which are reflective. 
This is expected to influence the results at low frequency, but since the sleepers dominate the radiated noise below about $400 \mathrm{~Hz}$ [1], it has been neglected up to now. In the current work, the effect of the proximity of the ground on the rail radiation is studied in terms of its radiation ratio. In order to propose a more realistic model to predict the noise from the rail at low frequencies, the effects of acoustic boundary conditions due to the sleeper and ballast are taken into account. Results are presented initially for the radiation ratio of the rail in different conditions (in free space, attached to a rigid ground and above a rigid ground) by using the boundary element method (BEM) in two dimensions (2D), for both vertical and horizontal vibration. Then numerical calculations are presented which investigate the effects on the sound radiation of an absorptive ground in close proximity to the rail. Allowance is made for the effect of wave propagation along the rail by applying a wavenumber correction in the $2 \mathrm{D}$ modelling. Finally, the numerical results are verified by comparison with experimental results obtained using a 1/5 scale rail model in different configurations.

\section{Sound radiation from the rail in two dimensions}

The sound radiation from the rail is presented here in terms of the radiation ratio, or radiation efficiency, which can be written as

$$
\sigma=\frac{W}{\rho_{0} c_{0} S\left\langle v^{2}\right\rangle}
$$

where $\rho_{0}, c_{0}$ are the density of the air and the speed of sound, respectively, $W$ is the radiated

sound power of the structure, $S$ is its total surface area and $\left\langle v^{2}\right\rangle$ is the surface-averaged meansquare normal velocity. The quantities $\sigma, W$ and $v$ are functions of frequency. 
The geometry of a rail can be considered as two-dimensional, with the third dimension being effectively infinite. Then $W$ becomes the power per unit length and $S$ becomes the perimeter length. As the wavelength of rail vibration is generally large compared with the acoustic wavelength, the radiated sound field can be approximated as two-dimensional, allowing it to be calculated using a two-dimensional boundary element model. The validity of such an approach has been considered in Ref. 12 and shown to be valid in practical situations for a rail in situ above about $250 \mathrm{~Hz}$, giving results within approximately $2 \mathrm{~dB}$ of that for the three-dimensional case.

\subsection{Effects of a rigid ground on the sound radiation from the rail}

The sound radiation for a CEN60E1 rail is investigated here using a two-dimensional boundary element model based on the direct BEM approach [18] and written in Matlab. Three cases are considered, as shown schematically in Fig. 1. As well as a rail in free field, these are a rail attached to a rigid surface, and a rail at a certain distance above the rigid surface. The former case can represent a rail attached to a sleeper or track slab via a flexible pad. The latter case can be considered to represent the condition above the ballast, although initially the ground is considered to be acoustically hard. In this case, the sound radiation of the rail also depends on the distance of the base of the rail from the ground. In all cases the ground is assumed to be nonmoving. In all the calculations it is assumed, for simplicity, that the rail vibrates with a rigid body motion either vertically or horizontally, which is valid for frequencies below about $2 \mathrm{kHz}$ for vertical motion and about $1 \mathrm{kHz}$ for lateral motion. At higher frequencies, cross-sectional deformation will occur which is neglected here [12].

In order to include the ground in the model, a half-space formulation could be used. However, in order to allow for an absorptive surface later, a rigid box is included beneath the rail with 
dimensions chosen to be large enough that the edges have negligible effect on the results. This box has to be closed in the direct BEM method but apart from the top its surfaces are arbitrary. The box should extend at least one acoustic wavelength on each side of the rail. Two different models are used for low and high frequencies. The box dimensions are $20 \mathrm{~m}$ (width) $\times 1.05 \mathrm{~m}$ (height) for low frequencies up to $500 \mathrm{~Hz}$ and $3 \mathrm{~m} \times 0.1 \mathrm{~m}$ for high frequencies for the case of the rail on the ground. For the rail above the ground a $15 \mathrm{~m}$ wide box was used at low frequencies but the other dimensions were the same. The boxes are shown in Fig. 2. In each case the sound radiation from the rail will be normalised according to the radiating surfaces of the rail using Equation (1). As is well known, for exterior BE problems, singularities occur at the natural frequencies of the corresponding interior problem. To overcome this, the 'CHIEF' method [19] is used in which a small number of additional points are added inside the mesh to constrain the problem.

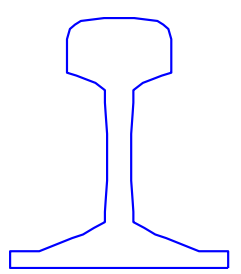

(a) In free space

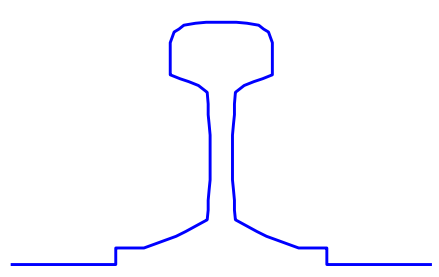

(b) On rigid ground

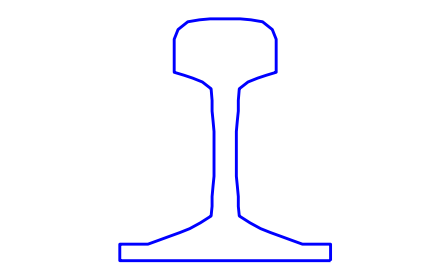

(c) Located above a rigid ground

Fig. 1. Two-dimensional Boundary Element models of a rail 


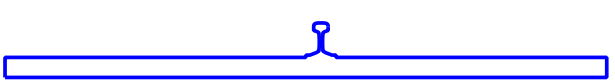

(a) On rigid ground

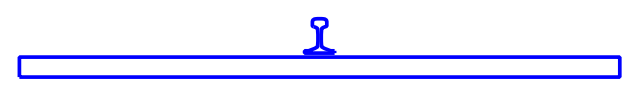

(b) Located above a rigid ground

Fig. 2. Schematic view of box geometry in the modelling (high frequency mesh)

In the BEM calculation, 87 elements are used to represent the rail with a maximum element size of $0.01 \mathrm{~m}$, which means there are at least 7 elements per wavelength at the maximum frequency of $5 \mathrm{kHz}$ used in the prediction. For the ground box, the maximum element size is $0.015 \mathrm{~m}$ at high frequency and $0.1 \mathrm{~m}$ at low frequency, corresponding to one quarter of the smallest wavelength in the corresponding frequency range. Fig. 3 shows the radiation ratio of the rail predicted for the three cases shown in Fig. 1. For both vertical and lateral motion, Fig. 3 shows that the radiation ratio of the rail in free space is proportional to $f^{3}$ at low frequency, which is characteristic of a line dipole. For vertical motion, above around $1 \mathrm{kHz}$ the radiation ratio oscillates around unity. Peaks and dips are exhibited which are due to the shape of the rail. Constructive and destructive interference between the sound radiation from the rail head and the rail foot cause these fluctuations in the radiation ratio. Taking the dip at $1 \mathrm{kHz}$, for example, the distance between the centre of the rail head and the rail foot is approximately half the acoustic wavelength at this frequency. Thus a dip occurs at this frequency because of destructive effects, whereas at $2 \mathrm{kHz}$ the distance is equal to the wavelength and a peak occurs.

When the rail is located above the rigid ground, the line dipole source will be reflected in the ground plane, so that for vertical motion a line quadrupole will be formed by the source and its image. This gives a slope proportional to $f^{5}$ at low frequency. It also can be seen that a peak occurs at $850 \mathrm{~Hz}$ when the rail foot is $0.02 \mathrm{~m}$ above the ground. At this frequency the acoustic 
wavelength is equal to $0.4 \mathrm{~m}$ which is twice the distance between the centre of the rail and its image source; hence at this frequency the two dipoles reinforce. For larger distances between the rail and the ground the peak moves to lower frequencies and becomes less pronounced. For the model of the rail attached to the rigid ground, however, the bottom of the rail foot cannot radiate sound, which means that the top and the bottom areas of the rail section are not equal. A net line monopole is present and the radiation ratio is proportional to $f$ at low frequency. At high frequencies the results are similar for all three cases. However, a sharp peak occurs when the distance between the rail foot and the ground is equal to half a wavelength $(3.4 \mathrm{kHz}$ for $50 \mathrm{~mm}$ separation, $1.7 \mathrm{kHz}$ for $100 \mathrm{~mm}$ ) as a narrow cavity is formed beneath the rail foot.

Fig. 3(b) shows the corresponding results when the rail vibrates laterally. At high frequencies, the radiation ratio again tends to unity, although different peaks and dips occur for the three cases due to the constructive and destructive interference effects of the different parts of the rail. At low frequencies the calculated radiation ratios in these three cases have the same slope, which is again proportional to $f^{3}$. Therefore, when the rail moves laterally, the rail source can be treated as a line dipole for all three cases. However, in the presence of the rigid ground the effective size of the dipole is increased, shifting the transition frequency downwards and increasing the radiation ratio at low frequencies. 


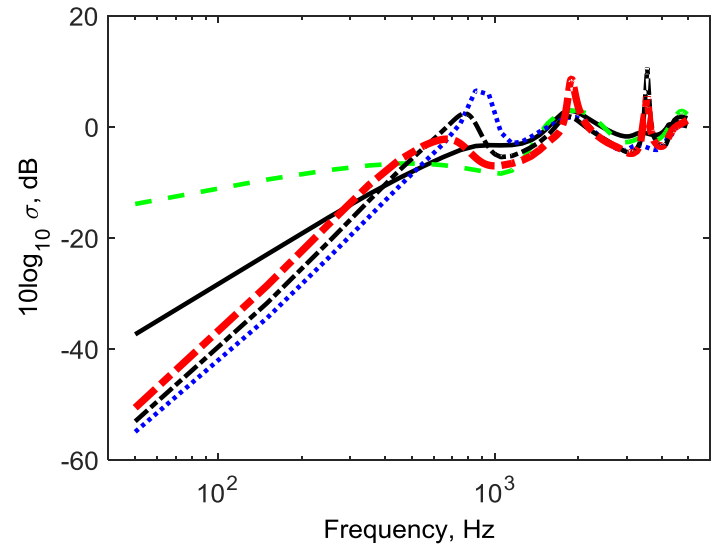

(a) Vertical motion

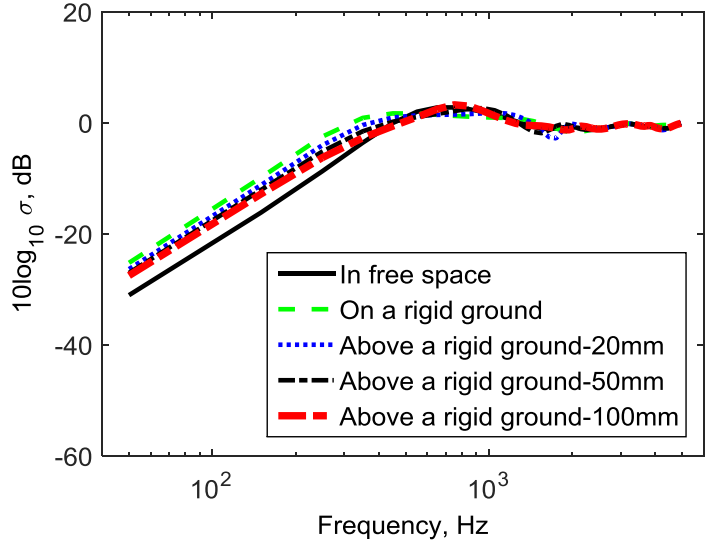

(b) Lateral motion

Fig. 3. Sound radiation of the rail calculated for free space and in presence of a rigid ground

\subsection{Effects of an absorptive ground on the sound radiation of the rail}

In practice, the ground is not rigid, but has absorptive properties which can be described by the ground impedance. For engineering outdoor sound propagation problems, a simple and widely used model for ground impedance is that originally proposed by Delany and Bazley [20] for porous materials. This model depends only on a single parameter, the flow resistivity, $\sigma_{e}$.

$$
z_{n}^{\prime}=1+9.08\left(\frac{1000 f}{\sigma_{e}}\right)^{-0.75}-11.9 i\left(\frac{1000 f}{\sigma_{e}}\right)^{-0.73}
$$

where $z_{n}=z_{n} / \rho_{0} c_{0}$ with $z_{n}$ the normal specific acoustic impedance of the ground and $\rho_{0} c_{0}$ the specific impedance of air. This form of the equation applies for flow resistivity in Pa.s $/ \mathrm{m}^{2}$.

For ground, an equivalent value of flow resistivity can be selected by comparison with a measured impedance. Although more elaborate models are also available, e.g. [21], for simplicity in the present study the Delany and Bazley model is used with a range of values of flow resistivity in order to test the sensitivity of the results to variations in the ground impedance. Fig. 4 shows the ground impedances obtained with this model for different values of flow 
resistivity for an infinitely thick layer of material. As can be seen, the normalised impedance $z_{n} / \rho_{0} c_{0}$ tends to 1 as frequency increases, but at low frequency it has large real and (negative) imaginary parts. It can be expected that as the normalised impedance tends to 1 , the surface will be able to absorb sound effectively. As shown in Fig. 4, the impedances obtained using the lower values of flow resistivity correspond to a more absorptive ground.

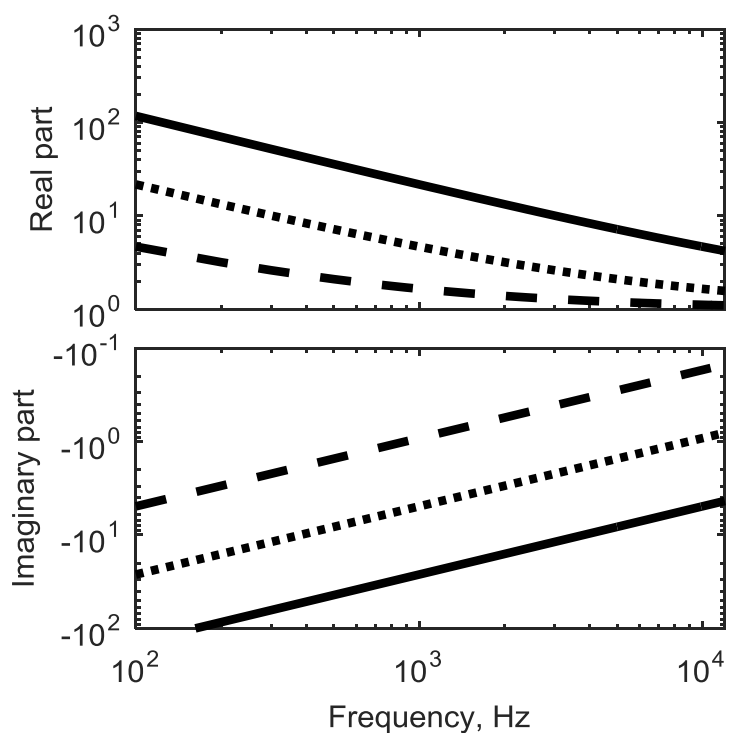

Fig. 4. Normalised impedances used for the ground obtained using different values of flow resistivity in Pa.s.m ${ }^{-2} .-3 \times 10^{6} ; \cdots \cdot 3 \times 10^{5} ;--3 \times 10^{4}$

This simple model for the impedance of the ground surface has been introduced into the boundary element simulations. Fig. 5 shows the calculation results for the rail attached to the ground undergoing both vertical and lateral motion. Results are shown for three values of flow resistivity for an infinite layer of absorptive material; the corresponding impedances are shown in Figure 4. As can be seen in Fig. 5, for both vertical and lateral motion, the rail will radiate less noise when attached to an absorptive ground than for a rigid ground, especially at low frequency.

Likewise, the sound radiation of the rail located $0.02 \mathrm{~m}$ above an absorptive ground is shown in Fig. 6. It can be seen in Fig. 6(a) that for vertical motion a more absorptive ground causes the rail 
to radiate more noise at low frequency. This is because the absorptive ground partly destroys the cancellation present for a reflective ground which led to the formation of a quadrupole source. At higher frequency, however, particularly between 600 and $2000 \mathrm{~Hz}$, the absorptive ground causes a reduction in the sound radiation of the rail. On the other hand, for lateral motion, as the ground becomes more absorptive the rail radiates less noise over the whole frequency range, especially between 200 and $1000 \mathrm{~Hz}$, where reductions of up to $7 \mathrm{~dB}$ are obtained.

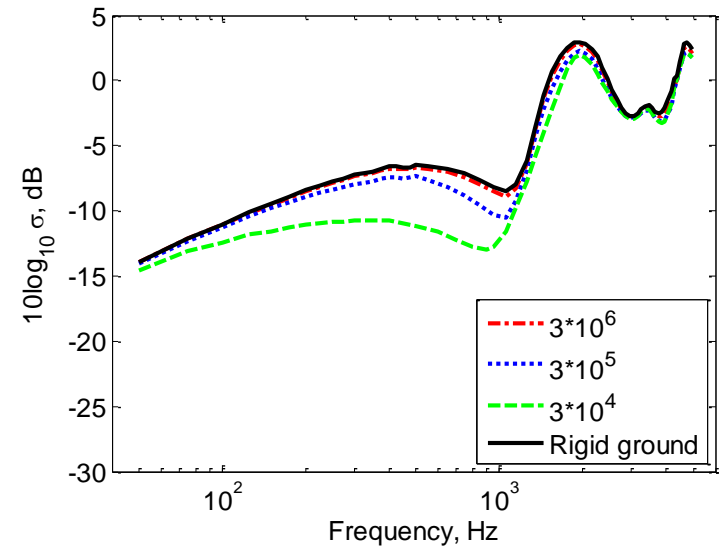

(a) Vertical motion

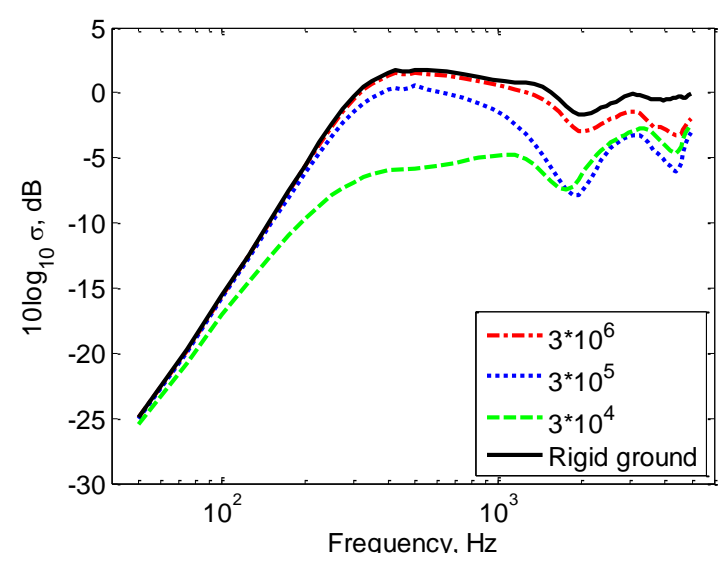

(b) Lateral motion

Fig. 5. Sound radiation of the rail attached to an absorptive ground with different values of flow resistivity (in Pa.s.m ${ }^{-2}$ )

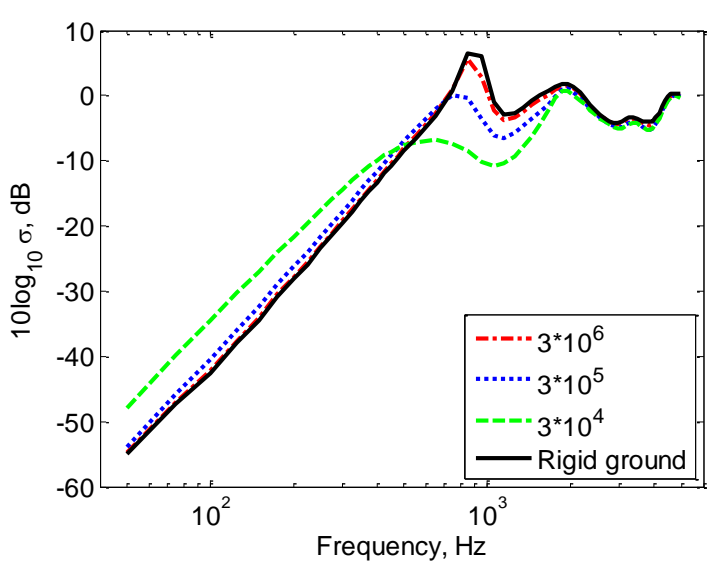

(a) Vertical motion

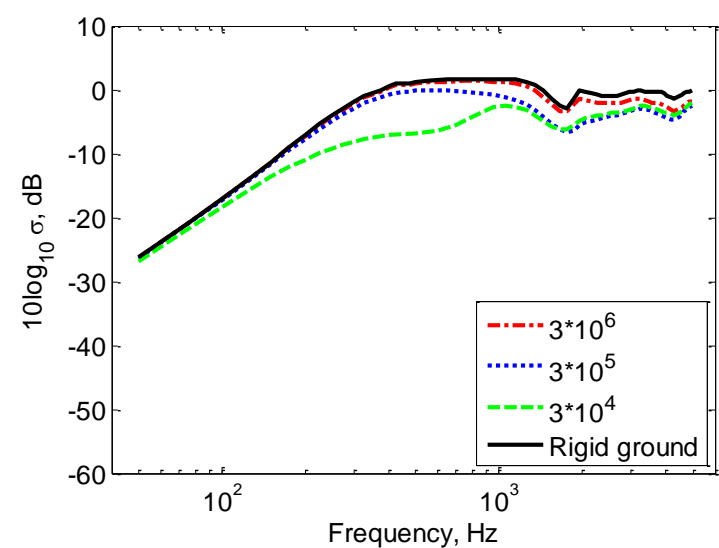

(b) Lateral motion 
Fig. 6. Sound radiation of the rail located $0.02 \mathrm{~m}$ above an absorptive ground with different values of flow resistivity (in Pa.s.m ${ }^{-2}$ )

\section{Sound radiation of the rail allowing for wave propagation}

\subsection{Wavenumbers in a rail}

The numerical predictions described above are based on the rigid body motion of the rail crosssection in two dimensions. This assumption is valid if the wavenumber in the rail is much smaller than the wavenumber of sound in air [12]. It is no longer valid at low frequency, especially for an unsupported rail for which a critical frequency $f_{c}$ exists, at which the wavenumber in the rail is equal to that in air.

In order to obtain the wavenumbers of a rail for different wave types, a 2.5D finite element method has been applied. WANDS [13], a 2.5D FE software, is used here to extract the wavenumber of an unsupported rail. This method allows the eigenvalue problem to be solved to obtain the frequencies corresponding to specified wavenumbers. Fig. 7(a) presents these dispersion results for the unsupported rail vibrating in vertical/longitudinal waves, while Fig. 7(b) gives the results for lateral/torsional waves. As can be seen, for both vertical and lateral motion, various wave types occur. More details of the various waves can be found in [13,22]. The first wave type in each case is the bending wave. Also shown in Fig. 7 is the acoustic wavenumber. The frequency at which this line crosses the bending wave curve can be identified as the critical frequency. For vertical motion this is $57 \mathrm{~Hz}$; for lateral motion it is $141 \mathrm{~Hz}$. 


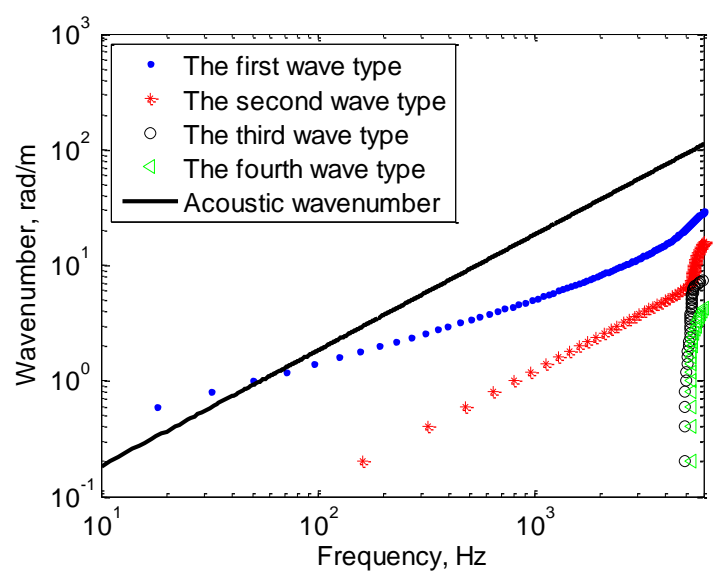

(a) Vertical motion

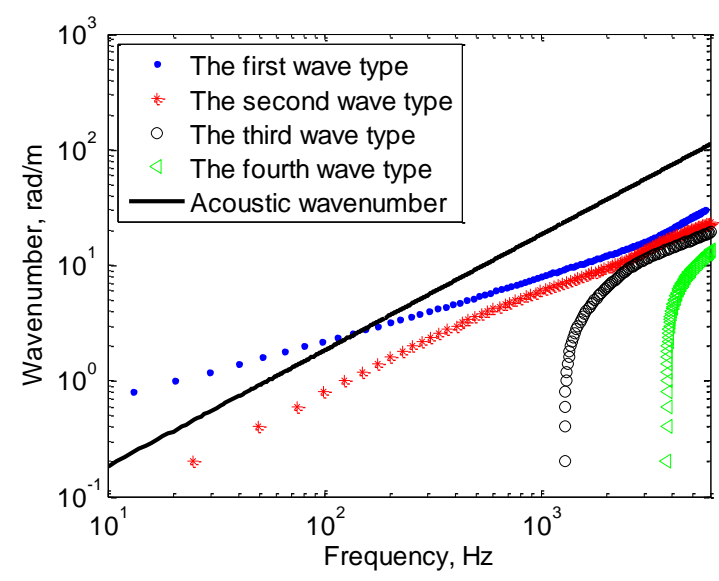

(b) Lateral motion

Fig. 7. Structural wavenumbers of waves in an unsupported rail

In practice the rail is supported on rail pads. A 2.5D FE model of a supported rail has also therefore been established in the WANDS software. The stiffness of the support is taken as $200 \times 10^{6} \mathrm{~N} / \mathrm{m}^{2}$ in the vertical direction and $77 \times 10^{6} \mathrm{~N} / \mathrm{m}^{2}$ in the lateral direction corresponding to a soft rail pad. This model corresponds to a continuous support beneath the rail; no damping is included. Fig. 8 presents the corresponding dispersion curves for both vertical and lateral motion. As can be seen, the various wave types in the supported rail cut on at a non-zero frequency. For the currently used values of support stiffness the vertical bending wave cuts on at $327 \mathrm{~Hz}$ and the lateral bending wave at $108 \mathrm{~Hz}$. Compared with the results for the unsupported rail, there are no critical frequencies; all wavenumbers are lower than the corresponding acoustic wavenumber. For a stiffer support the cut-on frequencies would be higher and again there would be no critical frequency. 


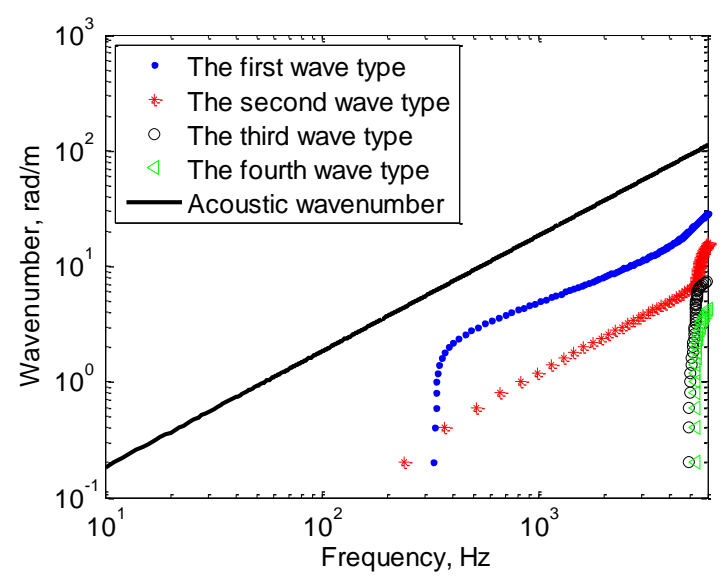

(a) Vertical motion

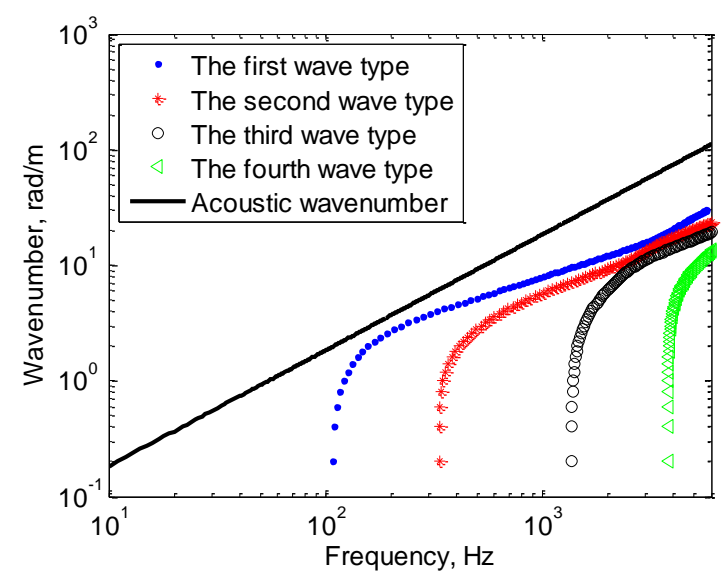

(b) Lateral motion

Fig. 8. Structural wavenumbers of waves in the supported rail

\subsection{Corrections to sound radiation}

When a wave propagates along the rail, it has a wavenumber $k_{x}$ as shown in Fig. 7 and 8. For an infinite rail with low decay rate, the corresponding sound field will share this wavenumber in the axial $(x)$ direction along the rail. The radiation within the $y-z$ plane, perpendicular to the rail, should therefore be modified to use a wavenumber $k_{2 D}$ given by [23]

$$
k_{2 D}=\sqrt{k^{2}-k_{x}^{2}}
$$

where $k=\omega / c_{0}$ is the acoustic wavenumber at circular frequency $\omega$.

The 2D BEM calculations presented in section 2 are based on the assumption that $k_{x} \square k$ and hence $k_{2 D} \approx k$. When $k_{x}=k$, however, $k_{2 D}=0$; the corresponding frequency is the critical frequency $f_{c}$. Below the critical frequency, $k_{2 D}$ is imaginary. 
For frequencies below around $2 \mathrm{kHz}$, the bending wave dominates in the structural vibration of the rail [1]. The wavenumber ratio $k_{2 D} / k$ for vertical and lateral bending waves in an unsupported rail up to $2 \mathrm{kHz}$ is presented in Fig. 9(a). The critical frequency is marked by the vertical dotted and dashed lines for vertical and lateral motion, respectively. As can be seen, the ratio $k_{2 D} / k$ increases from 0 at $f_{c}$ and tends to 1 at high frequencies.

For the prediction of the radiation from the unsupported rail at low frequency, it is necessary to use a modified wavenumber in the $2 \mathrm{D} \mathrm{BE}$ calculation, according to Eq. (3). This modified wavenumber will be used in the boundary element model to calculate the rail radiation for frequencies above the critical frequency.

For a supported rail, however, no waves propagate below the cut-on frequency of waves in the rail. Below that frequency the waves are strongly attenuated and a point source model is more appropriate than the 2D line source model [12]. The corresponding wavenumber ratio for the supported rail is presented in Fig. 9(b), which is different from the results for the unsupported rail at low frequencies. For the vertical motion, the wavenumber ratio is close to 1 over the whole frequency range above the cut-on frequency at $327 \mathrm{~Hz}$. This means that the 2D numerical prediction for the sound radiation without wavenumber correction will be valid for the supported rail above the corresponding cut-on frequency.

For the lateral direction, as can be seen, the cut-on frequency of the supported rail is lower than for the vertical direction due to the lower stiffness of the rail pad and the lower bending stiffness of the rail. In addition, a higher rail pad of $225 \mathrm{MN} / \mathrm{m}^{2}$ is also considered for the lateral direction. This gives a cut-on frequency of $173 \mathrm{~Hz}$. The ratio $k_{2 D} / k$ has minimum values of 0.67 for the soft pad and 0.8 for the stiff one. 


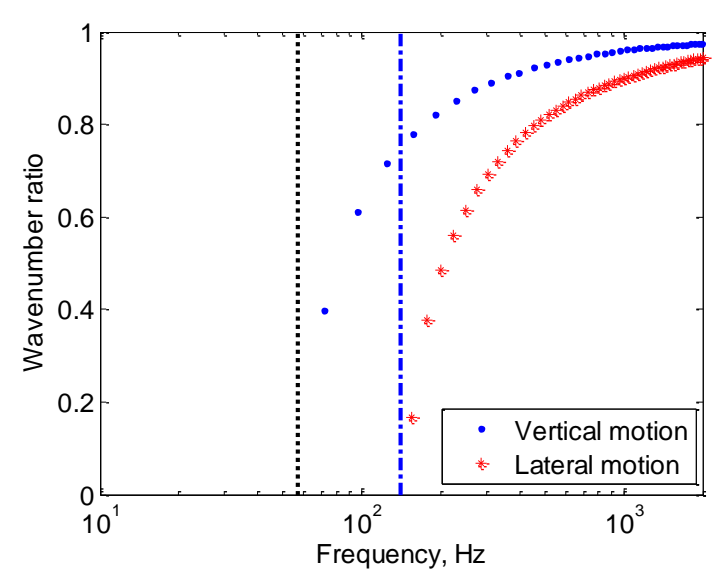

(a) Unsupported rail

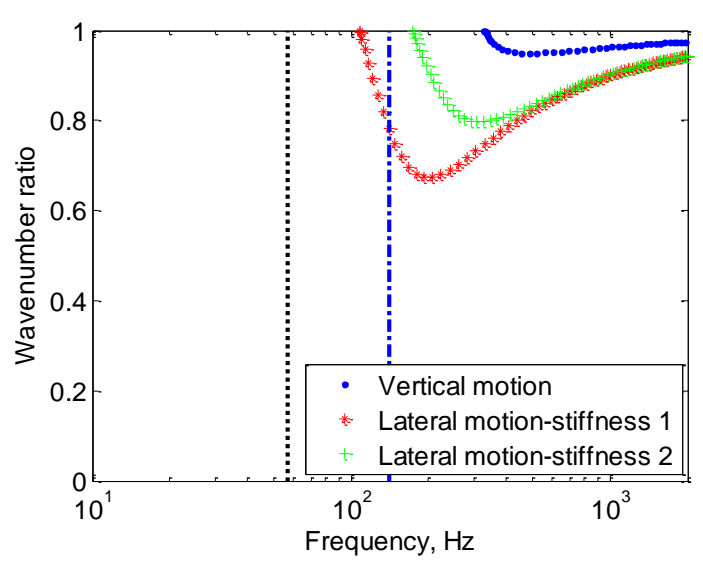

(b) Supported rail

Fig. 9. Acoustic wavenumber ratio $k_{2 D} / k$

Fig. 10 shows a comparison of the 2D numerical prediction of the radiation of an unsupported rail in close proximity to the rigid ground with and without the wavenumber correction. As can be seen, for both vertical and lateral motion, the corrected 2D prediction drops at low frequency, especially close to the corresponding critical frequencies. At high frequency, however, the radiation ratios of the rail with and without correction are nearly the same as each other.

Comparison is also made with the sound radiation of the supported rail for the free space boundary conditions in Fig. 11. As can be seen, for vertical motion, the sound radiation from the supported rail is identical to that for the unsupported rail. No corrections are necessary for the supported rail. For lateral motion, the sound radiation from the supported rail with both soft and stiff rail pads is predicted, as shown in Fig. 11(b). It can be seen that with the rail pads the radiation ratio increases at low frequency, tending to the $2 \mathrm{D}$ result, so the corrections found for the unsupported rail will not be applicable for the supported rail at low frequency. Between approximately 250 and $500 \mathrm{~Hz}$ the radiation ratio of the supported rail is up to $5.6 \mathrm{~dB}$ lower than that obtained using the $2 \mathrm{D}$ analysis for the soft pad and $3.5 \mathrm{~dB}$ for the stiffer pad. This means 
that the $2 \mathrm{D}$ numerical correction in the lateral case at low frequency depends on the rail pad stiffness. At higher frequency, however, the 2D numerical prediction without wavenumber correction can be used for the lateral motion of the supported rail.

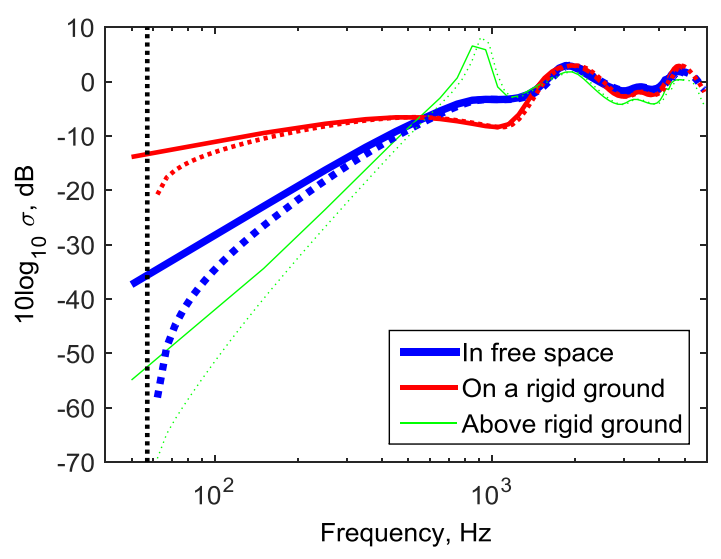

(a) Vertical motion

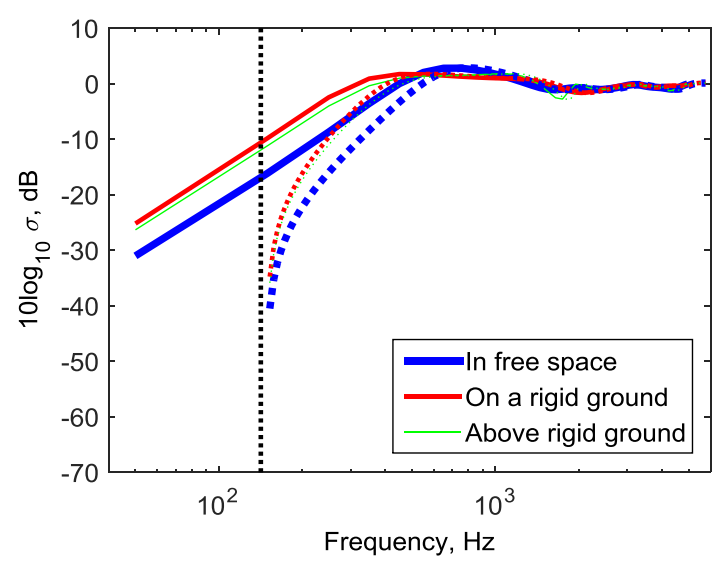

(b) Lateral motion

Fig. 10. Effects of a rigid ground on the sound radiation of the rail. Solid lines: 2D prediction; dotted lines: corrected 2D prediction

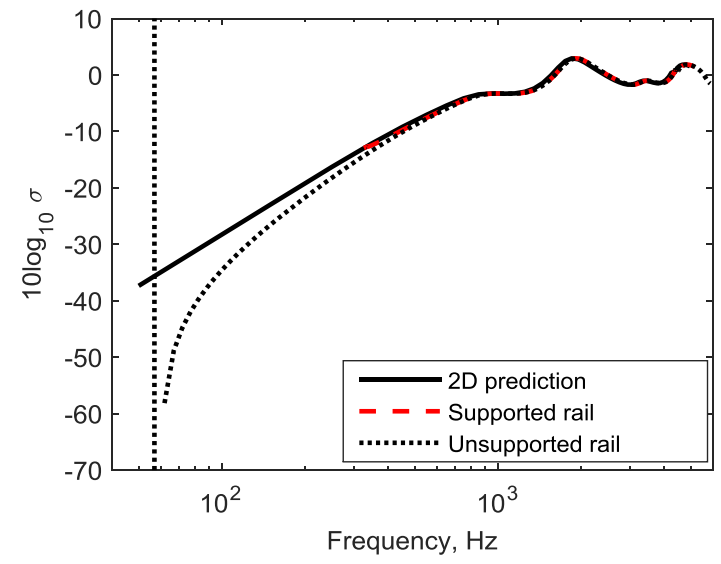

(a) Vertical motion

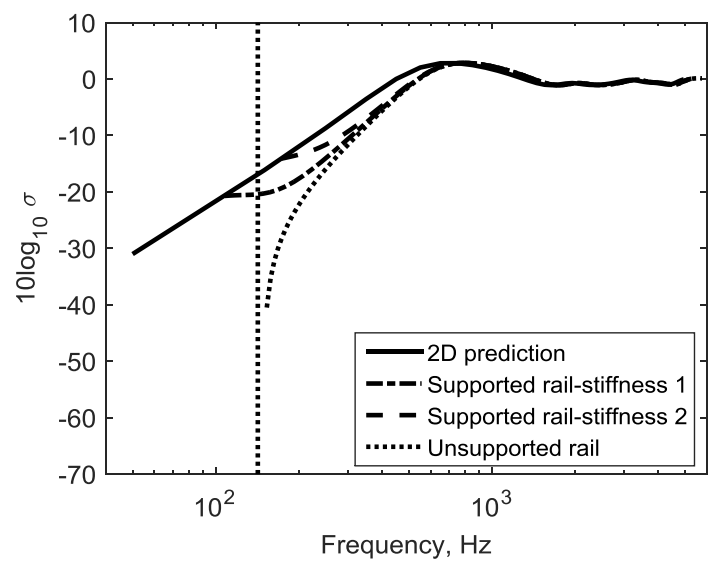

(b) Lateral motion

Fig. 11. Comparison of sound radiation of the supported rail and unsupported rail in free

$$
\text { space }
$$

The corresponding results for the rail with an absorptive ground with a flow resistivity of $3 \times 10^{5}$ Pa.s.m ${ }^{-2}$ are shown in Fig. 12 for the unsupported rail. Again, when the rail moves either 
vertically or laterally, the $2 \mathrm{D}$ numerical prediction overestimates the rail radiation at low frequency, but gives reasonable results at high frequency.

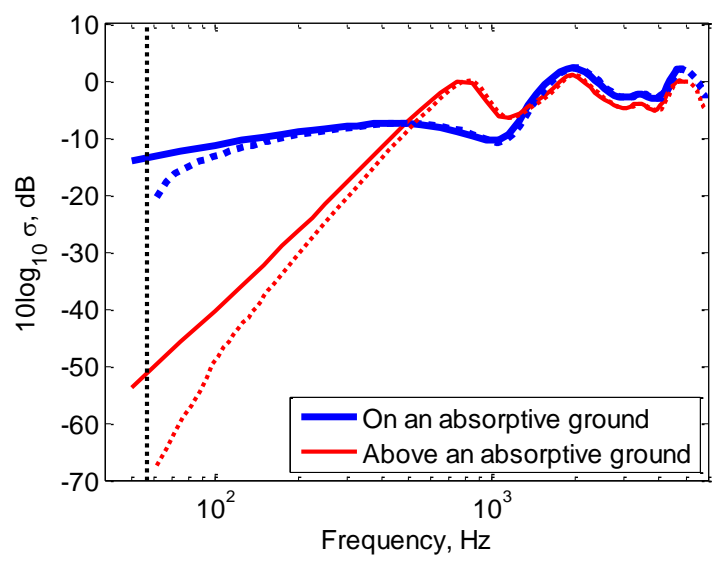

(a) Vertical motion

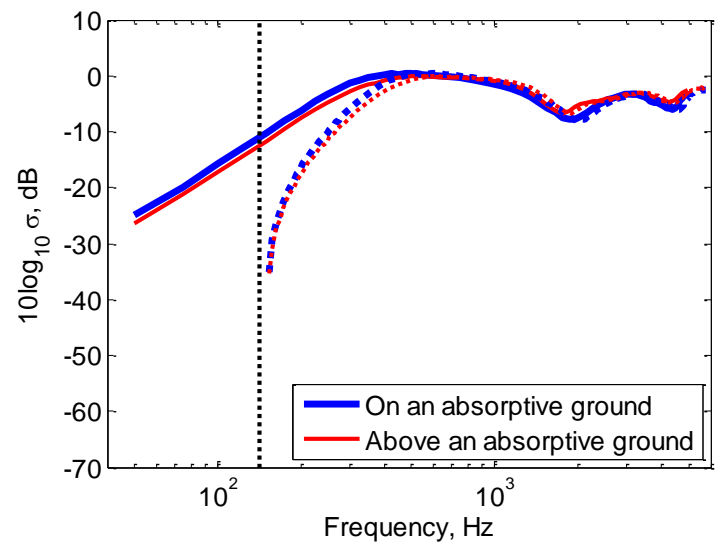

(b) Lateral motion

Fig. 12. Effects of an absorptive ground on the sound radiation of the rail. Solid lines: $2 \mathrm{D}$ prediction; dotted lines: corrected 2D prediction

\section{Experimental validation}

To validate the BEM modelling of the rail radiation described above, measurements have been carried out using a $1 / 5$ scale freely suspended rail. The rail has a length of $2 \mathrm{~m}$. It is a commercially available steel rail used for miniature railways and is approximately a $1 / 5$ scale replica of the CEN60E1 section. The dimensions of the full size and scale rail cross-sections are presented in Fig. 13. Note that the web is thicker than its full size counterpart and the head is slightly wider but otherwise the main dimensions are correct to a scale 1:5. The behaviour of this scale rail is therefore approximately that of a full size rail at 5 times the frequency. Thin strips of a constrained layer damping treatment with a thickness of $1 \mathrm{~mm}$ have been attached to the bottom of the rail foot. This increases the damping ratio of the rail and simplifies testing by 
reducing the dynamic range of the frequency response. Overall, the damping treatment increases the damping ratio to an average value of around 0.013 .

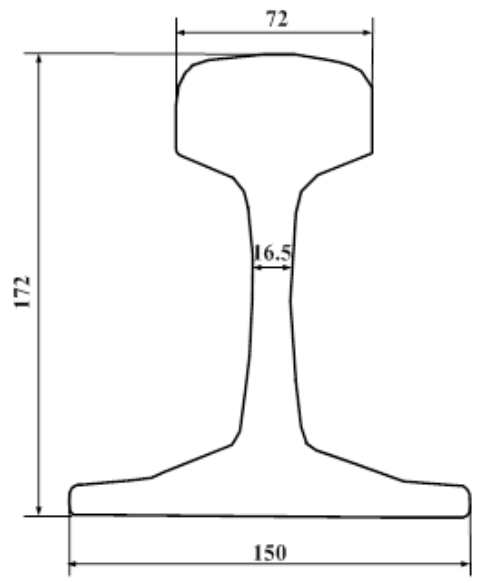

(a) Full size rail

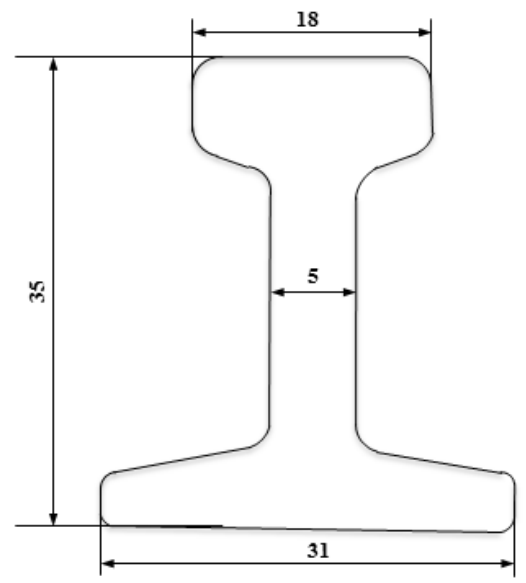

(b) $1 / 5$ scale rail

Fig. 13. Comparison of rail cross-section: dimensions in $\mathrm{mm}$

\subsection{Theory of experimental method}

The radiation ratio has been measured by adopting a reciprocity approach [24] in which the sound power is measured in a reverberant room by acoustically exciting the rail and measuring its vibration levels. The rail transfer mobility is measured separately. The validity of the reciprocity method has been checked against direct methods and numerical calculations for various test specimens in [24].

The approach is summarised here. Eq. (1) can be rearranged as

$$
\sigma=\frac{W / \overline{F^{2}}}{\rho_{0} c_{0} \sum_{i}\left|v_{i} / \bar{F}\right|^{2} d S_{i}}
$$


where $\frac{W}{\overline{F^{2}}}$ is the radiated sound power normalised by the mean-square force $\overline{F^{2}}$ and $\left|\frac{v_{i}}{\bar{F}}\right|^{2}$ is the squared mobility from the force position to a response position $i . d S_{i}$ is the surface area of the structure associated with position $i$.

According to Eq. (4), two tests are required. One is used to measure the transfer mobilities, while the other is used to obtain the sound power for a given squared force. The latter has been determined reciprocally by measuring the acceleration response to a sound pressure field in a reverberation chamber [24]. The sound power $W$ radiated by a vibrating structure, normalised by the mean-square force $\overline{F^{2}}$ exciting the structure, is equivalent to

$$
\frac{W}{F^{2}}=\frac{\rho_{0} \overline{a_{Q}^{2}}}{4 \pi c_{0}\left\langle p_{Q}^{2}\right\rangle}
$$

where $\overline{a_{Q}^{2}}$ is the mean-square acceleration of the structure at the original forcing position when excited acoustically and $\left\langle\overline{p_{Q}^{2}}\right\rangle$ is the spatially averaged mean-square pressure in the room. Note that it is not necessary to determine the reverberation time of the chamber as long as the sound field is diffuse.

\subsection{Test procedure}

The transfer mobility has been measured at 41 points, equally spaced along the rail, for three different excitation positions. A small impact hammer was used to allow the rail to be excited for frequencies up to $10 \mathrm{kHz}$. For each position of input force, the data obtained was averaged 5 times. Structural reciprocity has been used during the mobility tests, so that the hammer is 
moved along the length of the rail and the response is measured at the three fixed 'excitation' positions.

The acceleration response of the structure to a measured sound pressure field has been obtained in a reverberation chamber of volume $348 \mathrm{~m}^{3}$. Four loudspeakers, excited by white noise over the frequency range up to $10 \mathrm{kHz}$, were used to create the sound field. The spatially averaged sound pressure $\left\langle\overline{p_{Q}^{2}}\right\rangle$ has been obtained with a microphone placed on a rotating boom.

\subsection{Verification of rail sound radiation}

The scale rail was tested in free space, above a rigid ground and above an absorptive ground, for both vertical and lateral vibration. The case of the rail on a rigid ground, however, is not considered as it was found to be difficult to realize the same boundary conditions as in the BE model where the bottom of the rail foot was rigidly fixed to ground.

As the rails are tested in free condition structurally, it is necessary to determine the critical frequency $f_{c}$ of the scaled rail, which is the same as that defined for an infinite rail as shown in Eq. (3). A corresponding FE model has been made for the scale rail from which it is found that the critical frequency for vertical motion is $285 \mathrm{~Hz}$ for vertical motion and $700 \mathrm{~Hz}$ for lateral motion. The experimental results are compared with numerical predictions obtained for the scale rail in following sections.

\subsubsection{Rail in free space}

The rail was initially suspended by elastic cords $1 \mathrm{~m}$ above the floor of the chamber, which is far enough to consider the rail as being in free space for frequencies above $150 \mathrm{~Hz}$, at which the distance is more than half the acoustic wavelength (similarly in ISO 3741 [25] sources should be 
at least $1.5 \mathrm{~m}$ from the walls of the chamber for measurements above $100 \mathrm{~Hz}$ ). The rail acceleration was measured in the vertical direction in the centre of the rail head. A comparison of the measured sound pressure level with the background noise is shown in Fig. 14(a). All results are shown in one-twelfth octave bands. This provides some smoothing of the measured data, while retaining a finer resolution than the more usual one-third octaves. Fig. 14(b) shows equivalent results for the vertical acceleration signal. As can be seen, an adequate signal-to-noise ratio was achieved for the sound pressure, and at most frequencies above $300 \mathrm{~Hz}$ for the acceleration. Fig. 15(a) presents the normalised power obtained by the reciprocity method. The spatially averaged transfer mobility of the rail is shown in Fig. 15(b), which shows a series of peaks corresponding to the modes of the finite length of rail.

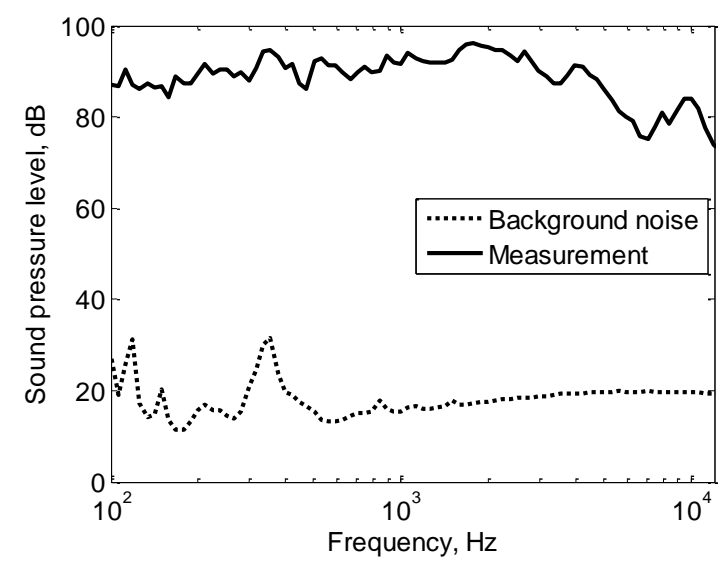

(a) Sound pressure level

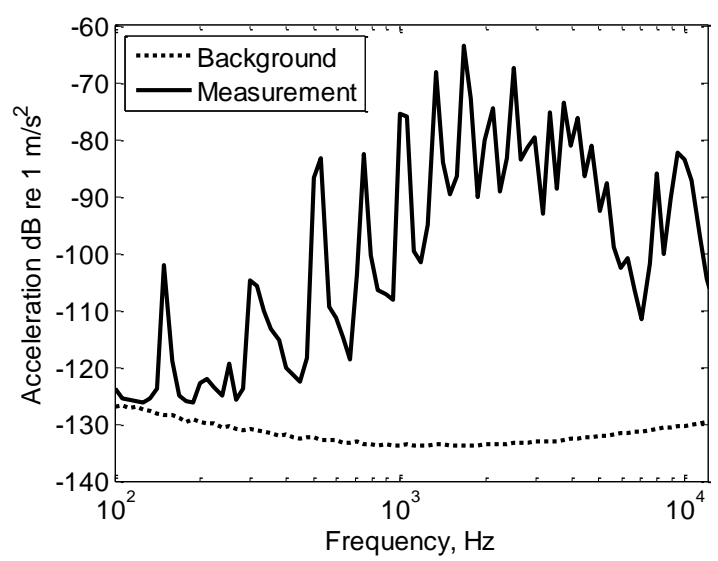

(b) Acceleration

Fig. 14. Comparison of measurement and background noise signals for rail in reverberation chamber: vertical motion in one-twelfth octave bands 


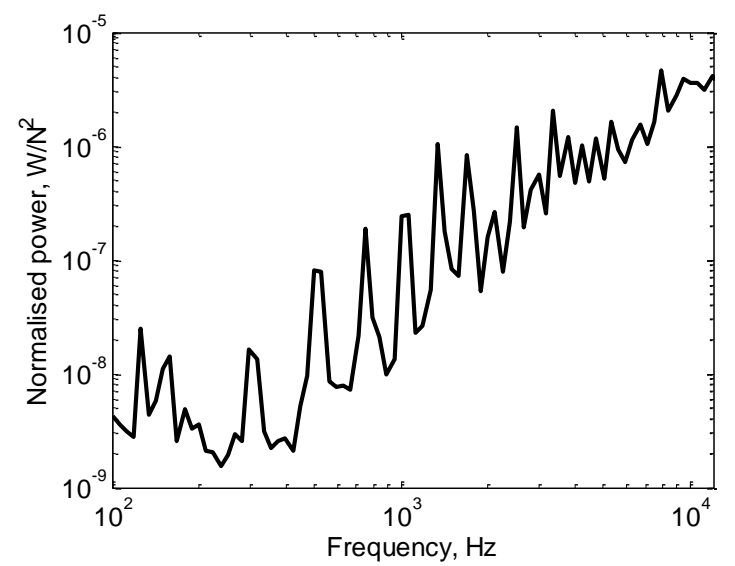

(a) Normalised power

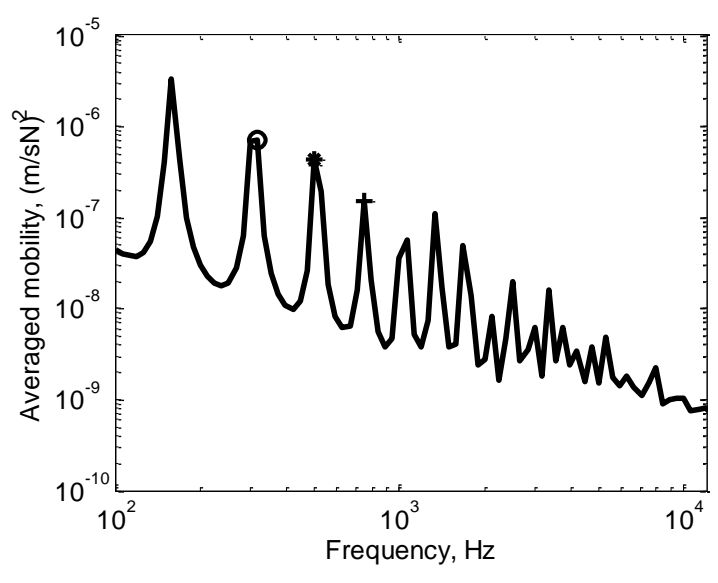

(b) Averaged mobility

Fig. 15. Normalised sound power and averaged mobility of $1 / 5$ scale rail in free space for vertical motion in one-twelfth octave bands

Fig. 16 (a) compares the numerical results and measurements for vertical motion of the rail in free space. These results correspond to a line dipole behaviour $\left(\sigma \sim f^{3}\right)$ at low frequency, rising to unity above $5 \mathrm{kHz}$ (or $1 \mathrm{kHz}$ for full scale). Above the critical frequency, it can be seen that the measurement curve has some peaks and dips between $300 \mathrm{~Hz}$ and $900 \mathrm{~Hz}$, not found in the numerical results. The largest dips, indicated by markers, correspond to modes of the rail, as also indicated in Fig. 14(b). Clearly the acoustic behaviour of these low frequency modes can differ from that of the simple line dipole even above the critical frequency. The measured results at the dips agree better with the predictions including the wavenumber correction. In the frequency range $900 \mathrm{~Hz} 4 \mathrm{kHz}$, the experimental results agree very well with the numerical predictions with or without correction. However, the measurement results are larger than the numerical ones above $4 \mathrm{kHz}$, probably because of the influence of cross-sectional deformation of the rail which occurs at high frequency, whereas the predictions are based on a rigid vertical motion of the rail. At around the critical frequency, the radiation ratio from the corrected $2 \mathrm{D}$ prediction drops dramatically. At and below the critical frequency, the measured result is strongly affected by the 
end effects of the finite length of the rail, which are not considered in the model. These are similar to corner and edge modes on a plate [23].

For the rail with lateral motion in free space in Fig. 16(b), the accelerometer positions were at the centre of the web, and the mobility was measured for excitation at the corresponding web position. This was used, instead of an excitation at the rail head, in order to minimise the contribution of torsional vibration of the rail and to give a better comparison with the boundary element results which are based on rigid lateral motion. As can be seen, good agreement is achieved up to $7 \mathrm{kHz}$. Between the critical frequency of $700 \mathrm{~Hz}$ and about $2 \mathrm{kHz}$ the measurement follows the trend of the corrected BEM predictions. At very high frequencies, the measured radiation ratio is greater than the predictions, which is again likely to be due to the neglect of cross-sectional deformation of the rail in the numerical model. Web bending is expected to occur at about $1.5 \mathrm{kHz}$ for the full scale rail [22], which corresponds to $7.5 \mathrm{kHz}$ for the $1 / 5$ scale rail. Below the critical frequency, the sound radiation will be again determined by end effects of the finite length rail.

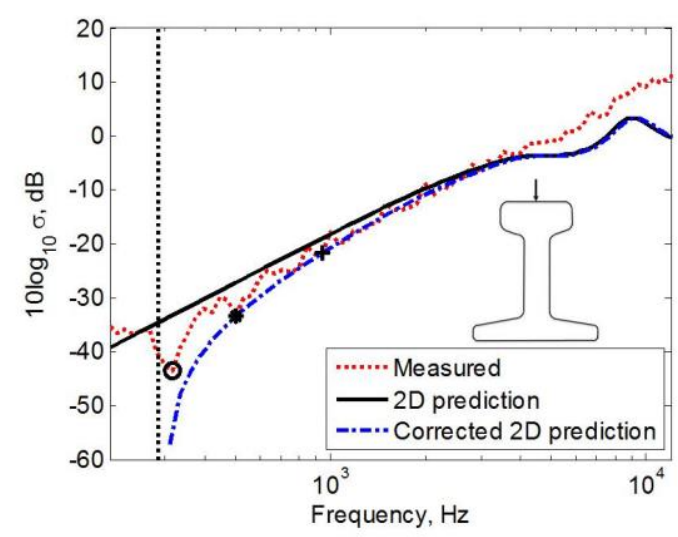

(a) Vertical motion

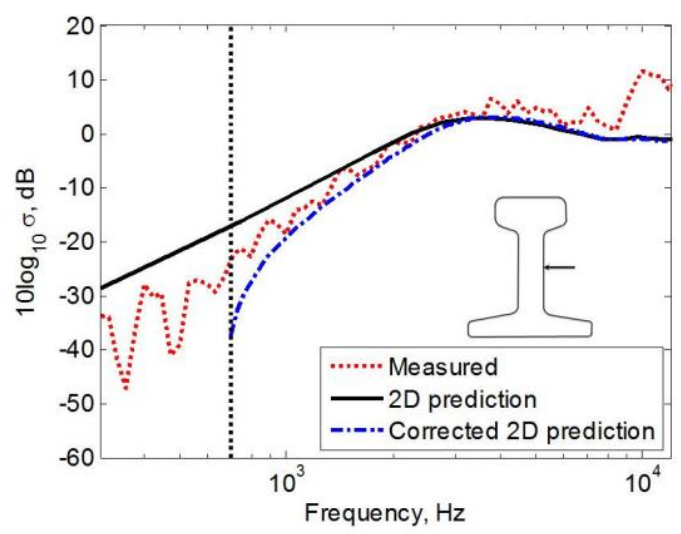

(b) Lateral motion

Fig. 16. Comparison of numerical results and measurements for $1 / 5$ scale rail in free space (the 
critical frequency is marked by a dotted vertical line)

\subsubsection{Rail above rigid ground}

The rail was next suspended $20 \mathrm{~mm}$ above the rigid floor of the chamber. It should be noted that the rail was not exactly straight and the ground is not completely flat either; therefore the distance was in the range $20 \mathrm{~mm} \pm 3 \mathrm{~mm}$. Fig. 17 presents the effect of introducing the ground in the form of the level difference between the radiation ratio of the rail above the rigid ground and that in free space. Results are given for the measurements and for the predictions with and without the wavenumber corrections.

For vertical motion, in Fig. 17(a), very good agreement can be seen between the experimental results and the numerical results, especially with the corrected 2D prediction. The markers correspond to the vibration modes indicated in Fig. 16(a). It can be seen that the scale rail will radiate more noise than that in free space between 1.5 and $3.5 \mathrm{kHz}$. The peak observed in the predictions at $9 \mathrm{kHz}$ is not seen in the measurements, possibly due to the variations in the distance above the ground.

In Fig. 17(b), for the rail vibrating laterally, the same trends are seen in the measurements and the numerical predictions and good agreement is seen, particularly between the experimental results and the corrected 2D prediction. Below $2 \mathrm{kHz}$, the level difference is greater than $0 \mathrm{~dB}$, which means the rail above the rigid ground will radiate slightly more noise than that in free space. 


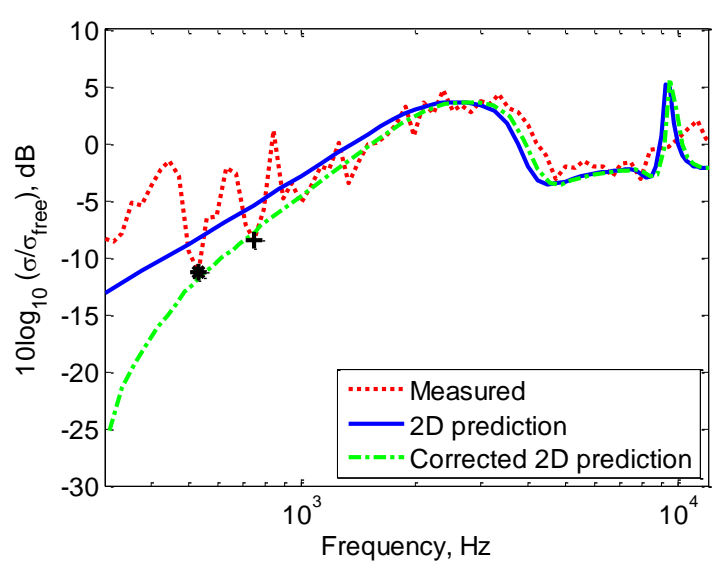

(a) Vertical motion

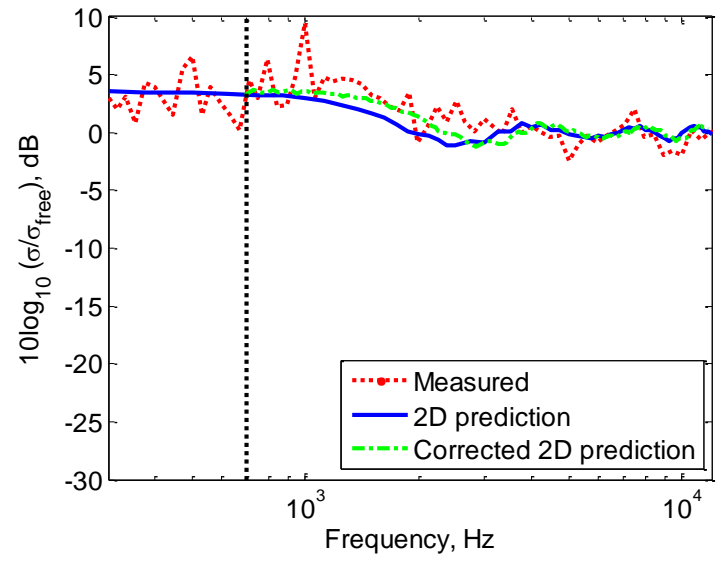

(b) Lateral motion

Fig. 17. Comparison of numerical results and measurements for $1 / 5$ scale rail $20 \mathrm{~mm}$ above rigid ground

\subsubsection{Rail above absorptive ground}

The effects of an absorptive ground on the sound radiation of the rail have been tested by using a layer of melamine foam, with dimensions $2 \mathrm{~m} \times 0.4 \mathrm{~m} \times 50 \mathrm{~mm}$. The corresponding set-up of the rail is presented in Fig. 18. Its non-dimensional surface normal impedance was measured in an impedance tube and an equivalent value of the flow resistivity has been deduced as $8000 \mathrm{~Pa} . \mathrm{s} / \mathrm{m}^{2}$ by fitting the Delany-Bazley model [21] to this measurement. Fig. 19 shows the comparison with the measured impedance. It can be seen that the theoretically estimated results, the dotted lines, agree reasonably well with the measurement. Note that the presence of the foam in the chamber will modify the reverberation time but since this is not present in Eq. (5) this will not influence the sound power measurements. 


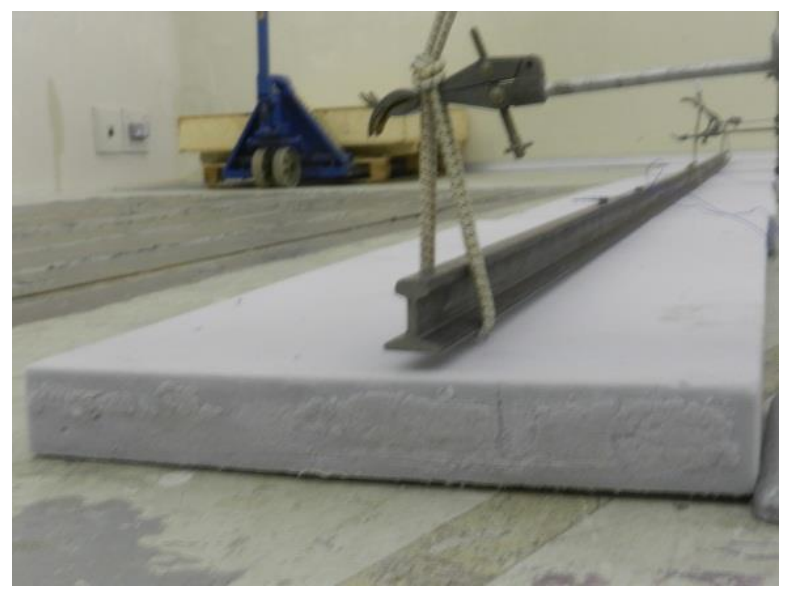

Fig. 18. Rail $20 \mathrm{~mm}$ above absorptive material

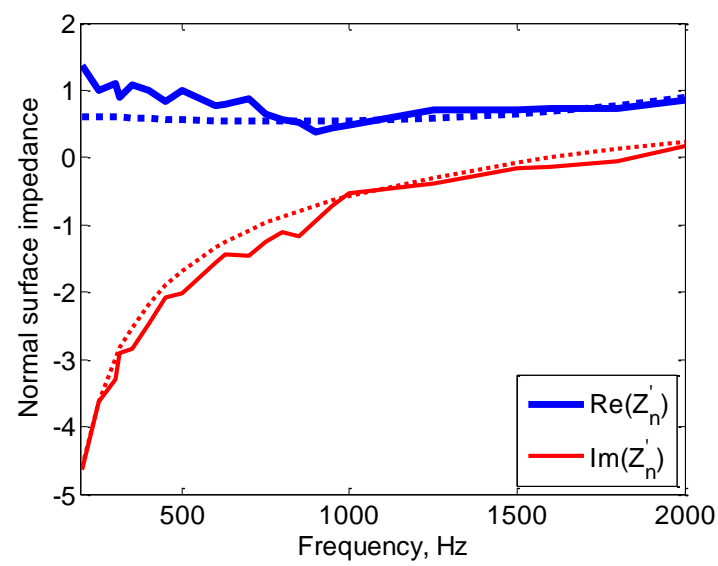

Fig. 19. Measured non-dimensional surface normal impedance for melamine foam $(-)$ and comparison with Delany-Bazley model (....)

A comparison of the measured radiation ratio with the numerical calculations is shown in Fig. 20. Again these results are normalised to the case of a rail in free space. Fig. 20(a) compares the measurement with the prediction for vertical motion. The measured results agree well with the predictions above $600 \mathrm{~Hz}$. Above $1500 \mathrm{~Hz}$ the absorptive ground causes the rail to radiate less noise than in free space. Fig. 20(b) shows the corresponding results for lateral vibration of the rail. Here the noise radiation is reduced by the presence of the absorptive ground for all frequencies above $900 \mathrm{~Hz}$. Similar trends are seen in the predictions. 


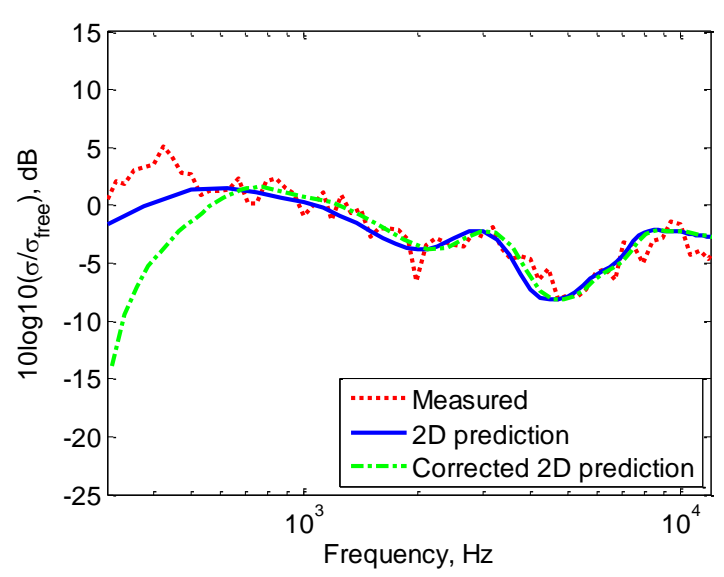

(a) Vertical motion

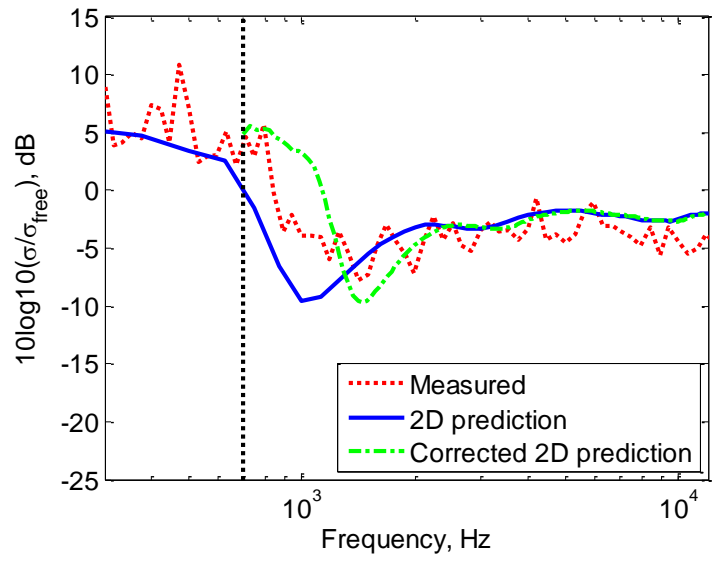

(b) Lateral motion

Fig. 20. Comparison of numerical results and measurements for $1 / 5$ scale rail $20 \mathrm{~mm}$ above absorptive ground

\section{Conclusions}

Using a two-dimensional boundary element model, it has been shown that the sound radiation of a rail in free space has the behaviour of a line dipole. For vertical motion above a rigid ground this is changed to that of a line quadrupole, while it becomes a line monopole when the rail is attached to the rigid ground. For lateral vibration of the rail, however, the radiation follows the characteristics of a line dipole for all three cases. Furthermore, if the rail is attached to an absorptive ground, the noise radiated by the rail reduces as the absorption is increased. For the rail above an absorptive ground, the ground absorption will reduce the sound radiated by lateral motion of the rail over the whole frequency range. For the rail vibrating vertically, however, at low frequency, an absorptive ground will make the rail radiate more noise than above a rigid ground but less than the rail in free space, whereas the rail radiation will be reduced at higher frequencies. 
Corrections to the $2 \mathrm{D}$ predictions have been introduced to allow for the wavenumber in an unsupported rail, which are significant in the vicinity of the critical frequency of the rail. However, for a supported rail in track these corrections are not applicable. For vertical motion it is shown that no correction is required whereas for lateral motion, the specific correction required at low frequency will be less than that for an unsupported rail and will depend on the stiffness of the rail pad used in practice.

Corresponding measurements have been performed on a reduced scale rail to verify the numerical predictions for the rail in different configurations. For both vertical and lateral vibration, very good agreement has been found between the measured results and numerical predictions for all configurations. In general, however, the measured radiation ratio is somewhat greater than the numerical estimates at high frequency, which is probably due to the presence of cross-section deformation which has been neglected in the BEM calculations.

\section{ACKNOWLEDGMENTS}

The work described has been supported by the EPSRC under the programme grant EP/H044949/1, 'Railway Track for the $21^{\text {st }}$ Century' (Track 21) and by the EU project ACOUTRAIN, Virtual certification of acoustic performance for freight and passenger trains, collaborative project number 284877 funded under the Seventh Framework. All data published in this paper are openly available from the University of Southampton repository at http://dx.doi.org/10.5258/SOTON/382992. 


\section{REFERENCES}

[1] D. J. Thompson. Railway noise and vibration mechanisms, modelling and means of control. Elsevier, Oxford, 2009.

[2] P.J. Remington. Wheel/rail noise-part IV: rolling noise. Journal of Sound and Vibration, 46, 419-436, 1976.

[3] P. J. Remington. Wheel/rail noise-part I characterization of the wheel/rail dynamic system, Journal of Sound and Vibration, 46, 359-380, 1976.

[4] E.K. Bender, P.J. Remington. The influence of rails on train noise. Journal of Sound and Vibration, 37, 321-334, 1974.

[5] P. J. Remington. Wheel/rail rolling noise I: theoretical analysis. Journal of the Acoustical Society of America, 81, 1805-1823, 1987.

[6] I. Chessell. Propagation of noise along a finite impedance boundary. Journal of the Acoustical Society of America, 62, 825-834, 1977.

[7] P. J. Remington. Wheel/rail rolling noise II: Validation of the theory, Journal of the Acoustical Society of America, 81, 1824-1832, 1987.

[8] D.J. Thompson. Wheel-rail noise generation, part 1 introduction and interaction model. Journal of Sound and Vibration, 161, 387-400, 1993.

[9] D.J. Thompson, B. Hemsworth, N. Vincent. Experimental validation of the TWINS prediction program for rolling noise, Part 1: description of the model and method. Journal of Sound and Vibration, 193, 123-135, 1996.

[10] D.J. Thompson, P. Fodiman, H. Mahé. Experimental validation of the TWINS prediction program for rolling noise, part 2: results. Journal of Sound and Vibration, 193, 137-147, 1996. 
[11] M.-F. Petit, M. Heckl, J. Bergemann, J. Baae. Calculation of the radiation efficiency of railway rails using multipole synthesis, Proceedings DAGA, 997-1000, 1992.

[12] D.J. Thompson, C.J.C. Jones, N. Turner. Investigation into the validity of twodimensional models for sound radiation from waves in rails. Journal of Acoustical Society of America, 113, 1965-1974, 2003.

[13] C.-M. Nilsson, C.J.C. Jones, D.J. Thompson and J. Ryue. A waveguide finite element and boundary element approach to calculating the sound radiated by railway and tram rails. Journal of Sound and Vibration, 321, 813-836, 2009.

[14] T. Kitagawa, D.J. Thompson. The horizontal directivity of noise radiated by a rail and implications for the use of microphone arrays. Journal of Sound and Vibration, 329, 202$220,2010$.

[15] X. Zhang, H. G. Jonasson. Directivity of railway noise sources. Journal of Sound and Vibration, 293, 995-1006, 2006.

[16] X. Zhang. The directivity of railway noise at different speeds. Journal of Sound and Vibration, 329, 5273-5288, 2010.

[17] C.J.C. Jones, D.J. Thompson. Extended validation of a theoretical model for railway rolling noise using novel wheel and track designs. Journal of Sound and Vibration, 267(3), 2003.

[18] T.W. Wu. Boundary element acoustics: Fundamentals and computer codes. WITpress, Southampton, 2000.

[19] H.A. Schenck. Improved integral formulation for acoustic radiation problem. The Journal of Acoustical Society of America, 44, 41-58, 1968. 
[20] M.E. Delany, E.N. Bazley. Acoustical Properties of fibrous absorbent materials. Applied Acoustics, 3, 105-116, 1970.

[21] J.F. Allard, N. Atalla. Propagation of Sound in Porous Media: modelling sound absorbing materials. Wiley, Chichester, 2009.

[22] D.J. Thompson. Experimental analysis of wave propagation in railway tracks. Journal of Sound and Vibration, 203, 867-888, 1997.

[23] F. Fahy, P. Gardonio. Sound and structural vibration: radiation, transmission and response. Second edition, Elsevier, Oxford, 2007.

[24] G. Squicciarini, A. Putra, D. J. Thompson, X. Zhang and M. A. Salim. Use of a reciprocity technique to measure the radiation efficiency of a vibrating structure. Applied Acoustics, 89, 107-121, 2015.

[25] Acoustics-Determination of sound power levels and sound energy levels of noise sources using sound pressure-Precisions methods for reverberation test rooms (ISO 3741:2010). International Standard Organisation. 